\title{
Análise do sistema de Mobile Payment implementado no transporte público na cidade de São Paulo
}

Solange Cristina Ricardo

Mestre em Administração pelo Programa de Pós-Graduação em Administração (PPGA) da Universidade Nove de Julho - UNINOVE. Membro do grupo de pesquisa CNPq GIANTI Grupo Interinstitucional de pesquisa sobre Adoção de Novas Tecnologias de Informação, São Paulo, Brasil solangecricardo@gmail.com

Henrique Mello Rodrigues de Freitas

Professor Pós-Doutor e Titular do PPGA/Uninove, líder da Linha Organizações e Empreendedorismo. Coordenador do grupo de pesquisa CNPq GIANTI - Grupo Interinstitucional de pesquisa sobre Adoção de Novas Tecnologias de Informação, Bolsista de Produtividade em Pesquisa do CNPq 1C, São Pauo, Brasil

freitas138@gmail.com

\section{Editor Científico: José Edson Lara}

Organização Comitê Científico

Double Blind Review pelo SEER/OJS

Recebido em 27.12.2015

Aprovado em 23.03.2017

Este trabalho foi licenciado com uma Licença Creative Commons - Atribuição - Não Comercial 3.0 Brasil 


\title{
Resumo
}

$\mathrm{Na}$ cidade de São Paulo, aproximadamente 10 milhões de pessoas utilizam transporte público diariamente. $O$ objetivo deste trabalho é apresentar 0 atual sistema de pagamento móvel implementado no transporte público na cidade de São Paulo, e as razões possíveis pelas quais esse sistema é muito pouco utilizado pelos usuários. É considerado como pagamento móvel qualquer pagamento em que um dispositivo móvel capaz de se conectar a redes móveis de comunicação é utilizado para iniciar, autorizar e confirmar uma transação comercial. A metodologia utilizada foi estudo de caso único e como resultados pode-se dizer que não há dúvidas de que o sistema de pagamento móvel implementado é uma novidade que facilita o dia a dia dos usuários. Porém, há deficiências a serem sanadas para que se torne um serviço atrativo e que incentive o usuário à adoção do mesmo.

Palavras-chave: Mobilidade; Pagamento móvel; Transporte público.

\section{Analysis of the Mobile Payment system implemented in public transport in the São Paulo City}

\begin{abstract}
In São Paulo city about 10 million people use public transport daily. The objective of this paper is to present the current mobile payment system implemented in public transport in the city of São Paulo, and the possible reasons why this system is very rarely used by users. The mobile payment is any payment in which a mobile device capable of connecting to mobile communication networks, is used to initiate, authorize and confirm a commercial transaction. The methodology used was single case study and as a result it can be said that there is no doubt that the mobile payment system implemented is a novelty that facilitates day-to-day users, however, there are deficiencies to be remedied so that become an attractive service and to encourage you to adopt the same.
\end{abstract}

Keywords: Mobility; Mobile payment; Public transport.

\section{Análisis del sistema de Pago Móvil implementado en transporte público en la ciudad de São Paulo}

\section{Resumen}

En São Paulo cerca de 10 millones de personas utilizan el transporte público todos los días. El objetivo de este trabajo es presentar el actual sistema de pago móvil implementado en el transporte público en la ciudad de Sao Paulo, y las posibles razones por las que este sistema es muy rara vez utilizado por los usuarios. El pago móvil es cualquier pago en el que un dispositivo móvil capaz de conectarse a redes de comunicaciones móviles, se utiliza para iniciar, autorizar y confirmar una transacción comercial. La metodología utilizada fue un estudio de caso y como resultado, se puede decir que no hay duda de que el sistema de pago móvil 
implementada es una novedad que hace que los usuarios del día a día, sin embargo, hay irregularidades que deben subsanarse para que convertido en un servicio atractivo y que anima al usuario a adoptar la misma.

Palabras clave: Movilidad; Pago móvil; Transporte público.

\section{Introdução}

O transporte público é o meio mais utilizado por milhões de pessoas para se deslocarem até o trabalho, estudo e a lazer. Especificamente na cidade de São Paulo, mais de 10 milhões de pessoas utilizam o transporte público e, em sua maioria, essas pessoas têm um smartphone à mão, seja para conferir e enviar emails, interagir nas redes sociais, trocar mensagens, realizar compras e efetuar pagamentos.

À medida que os dispositivos móveis foram amplamente adotados pelos indivíduos, as organizações começaram a aplicar esse tipo de tecnologia de diferentes maneiras. Uma delas é a utilização de sistemas de pagamento móvel, considerada uma inovação financeira, possibilitando realizar a prestação de serviços financeiros.

O crescimento da computação móvel e ubíqua foi impulsionado pela transformação do ambiente corporativo e foi um grande avanço, permitindo que dados fossem acessados em tempo real, em ambientes geograficamente dispersos, possibilitando maior mobilidade. Os sistemas de comunicações móveis revolucionaram a forma como as pessoas se comunicam, unindo comunicação e mobilidade (Kumar, Liu, Sengupta \& Divya, 2010).

O mobile payment foi criado para ser um substituto do pagamento em dinheiro e dos cartões no comércio móvel, tendo sua utilização cada vez mais disseminada em diversos países. Um dos tipos de mobile payment utilizados é o Near Field Communication (NFC), também conhecida como tecnologia de campo por aproximação (Leong, Hew, Tan \& Ooi, 2013).

O sistema de pagamento móvel em transportes públicos tem sido cada vez mais implementado em substituição ou como alternativa aos sistemas já utilizados e na opinião de Finzgar e Tebar (2011), a conveniência de ter um sistema eletrônico de pagamento móvel à disposição da população torna essa tarefa mais fácil, segura e sem custos adicionais para o usuário, o que justifica a elaboração deste trabalho. 
Nesse contexto, o presente relato técnico tem por objetivo responder à seguinte questão: mesmo com as facilidades e vantagens que oferece, por que o sistema de pagamento móvel no transporte público na cidade de São Paulo está tão aquém do que poderia ser um serviço facilitador à população? O presente relato tem por objetivo apresentar o atual sistema de pagamento móvel no transporte público na cidade de São Paulo.

O método utilizado neste trabalho foi o estudo de caso, que, segundo Yin (2015), permite ao investigador um aprofundamento em relação ao fenômeno estudado, favorecendo uma visão holística sobre os acontecimentos da vida real, destacando seu caráter de investigação empírica de fenômenos contemporâneos. $O$ trabalho está estruturado em seis partes, começando pela introdução, seguida pela seção 2, na qual é apresentado o referencial teórico; a seção 3 apresenta a metodologia utilizada na pesquisa; na seção 4 são apresentados a organização e o projeto estudado; a seção 5 é dedicada ao tipo de intervenção adotada no estudo, bem como é feita a análise dos resultados obtidos. Por fim, na seção 6 são feitas as conclusões.

\section{Referencial Teórico}

\subsection{Tecnologias móveis e sem fio, mobilidade e ubiquidade}

Tecnologias da informação móveis e sem fio (TIMS), são aquelas que, por meio de um dispositivo móvel, possibilitam realizar comunicações, fazer chamadas de voz, enviar mensagens de texto e executar aplicativos. A utilização dessas tecnologias traz diversos atributos da mobilidade ao contexto empresarial, como liberdade de movimento, conveniência, conectividade instantânea, personalização e acessibilidade. Os avanços nas tecnologias de redes sem fio e móveis, segundo Pierre (2001), geraram um novo paradigma de computação, chamado de computação móvel, e de acordo com esse conceito, os usuários que carregam aparelhos portáteis têm acesso a informações em tempo real, através de uma infraestrutura compartilhada, independente de sua localização física.

A tecnologia móvel e suas implicações vão além de uma maior flexibilidade na comunicação e compartilhamento de informações, uma vez que elas melhoram o processo de trabalho, melhoram e podem influenciar o desempenho financeiro das 
empresas, permitindo melhores serviços ao cliente e criando vantagens competitivas. As tecnologias de informação sem fio "são tecnologias de informação que envolvem o uso de dispositivos conectados a uma rede ou a outro aparelho por links de comunicação sem fio" (Saccol \& Reinhard, 2007, p. 179). Os dispositivos móveis e sem fio permitem que as organizações conduzam os negócios dentro e através das fronteiras organizacionais de forma cada vez mais eficaz (Sheng, Nah \& Siau, 2005).

Desde o início da década de 80, a tecnologia foi incorporada como estratégia competitiva de negócios das empresas. A relevância da telecomunicação móvel surgiu na década de 90, quando houve um grande investimento por parte das operadoras de telefonia móvel e um aumento significativo de clientes em todo o mundo. Esse crescimento, tanto de usuários quanto de investimentos, perdurou durante uma década e meia, sendo a maioria associada à transmissão de voz; posteriormente, as operadoras acrescentaram serviços de valor agregado. A inovação tecnológica é também um fator constante na indústria de telefonia célula: de um lado, está o aumento de dispositivos móveis adaptados para o uso da Internet e para a difusão de dados que permitem a mobilidade e, de outro, a inovação e os investimentos em infraestrutura de rede de tecnologia permitem o aumento da disponibilidade no fornecimento de dados (Ghezzi, Cortimiglia \& Frank, 2015).

O objetivo 'a qualquer hora, em qualquer lugar' da computação móvel é essencialmente uma abordagem reativa ao acesso à informação. A computação ubíqua é um grande conjunto de computação móvel, para além da mobilidade, que requere suporte para a interoperabilidade, escalabilidade, esperteza e invisibilidade, objetivando garantir que os usuários tenham acesso contínuo à computação sempre que precisarem (Saha \& Mukehejee, 2003).

\subsection{Pagamento móvel}

O pagamento móvel faz parte das chamadas tecnologias da informação móveis e sem fio (TIMS), criadas para serem utilizadas enquanto se está em movimento. Tais tecnologias, segundo Corso, Freitas \& Behr (2013, p. 6):

propiciam que os indivíduos exerçam diferentes papéis sociais, a qualquer hora, e em qualquer lugar. Ao permitir ao indivíduo se comunicar a qualquer momento e em qualquer lugar, a mobilidade muda a forma de os seres 
humanos interagirem, afetando suas relações sociais, familiares, afetivas e profissionais.

O pagamento móvel "é utilizado para iniciar, autorizar e confirmar uma troca de valor financeiro em troca de bens e serviços, e tem se tornado um foco importante para atividades comerciais e de pesquisa nos últimos anos"(Carr, 2007, p.1). pagamento móvel é aquele realizado através de um dispositivo móvel - celular, tablet sem fio, PDAs (Personal Digital Assistants) ou outro dispositivo que seja possível a conectividade com a rede de telefonia móvel - e que seja realizada a transação de um pagamento através da transferência do dinheiro do comprador ao recebedor (Ondrus \& Pigneur, 2007; Amoroso \& Watanabe, 2012; Carr, 2007).

A tendência é que os pagamentos móveis substituam o dinheiro, o cheque e os cartões. Através do dispositivo móvel, será possível, também, realizar diversos serviços que são feitos na rede bancária e pela Internet, como, por exemplo, pagamento de contas, transferência de fundos, entre outros (Kumar \& Rabara, 2010).

Para ser aceito no mercado, um sistema de pagamento móvel deve possuir algumas características, como 1) simplicidade: através de aplicações em que o cliente seja capaz de utilizá-lo da forma que lhe seja mais conveniente; 2) universalidade: através de pagamentos de cliente para outro cliente, de uma empresa para um cliente, entre empresas; 3) interoperabilidade: o sistema deve ser desenvolvido e implementado de forma a interagir com outros sistemas; 4) segurança, privacidade e confiança: o cliente deverá confiar em um provedor de aplicativo de pagamento móvel, através de operações confidenciais e privativas; 5) custo: deve ser compatível com os demais meios de pagamento existentes; 6) velocidade: aceitável tanto para comerciantes quanto para seus clientes; e por fim, para se tornar amplamente aceita, a aplicação do pagamento móvel deve estar 7) disponível: em todo o tempo, em todo lugar (Carr, 2007).

A utilização de sistemas de pagamento móvel pode ser considerada como uma das inovações mais promissoras para o desenvolvimento da inclusão financeira. Isso se deve ao crescimento das vendas de smartphones e a facilidade de uso de tecnologias móveis. Segundo dados da Federação Brasileira de Bancos (Febraban, 2015), o crescimento do número de usuários de Mobile Banking foi 2,7 vezes maior 
em 2013 em relação a 2012, e o volume de transação nesse canal também aumentou 223,4\% ao ano. Os cartões de crédito e débito aparecem como meio de pagamento altamente utilizado, indicando o intenso uso e importância da tecnologia neste canal. Diante desse cenário, em 2010 foi criado por essa Federação um modelo de pagamento móvel para o mercado brasileiro, conforme observa-se na Figura 1.

\begin{tabular}{|c|c|}
\hline Universalidade & $\begin{array}{l}\text { - nacional, multi-banco, multi-bandeira, } \\
\text { multi-operadora, multi-comprador, com } \\
\text { tecnologia que permita massificação }\end{array}$ \\
\hline Instantaneidade & $\begin{array}{l}\text { - produtos simples e rápidos, } 7 \text { dias por } \\
\text { semana, } 24 \text { horas por dia }\end{array}$ \\
\hline Inclusão social & $\begin{array}{l}\text { • inclusão e suporte de bancarização de } \\
\text { público que não possui acesso ao } \\
\text { sistema financeiro básico }\end{array}$ \\
\hline Fácil adoção & $\begin{array}{l}\text { - adequação à cultura local, realidade } \\
\text { dos estabelecimentos comerciais e ao } \\
\text { perfil do usuário, facilitando o seu uso] }\end{array}$ \\
\hline Rápida implantação & $\begin{array}{l}\text { - disponibilidade no curto prazo, } \\
\text { integração aos meios de pagamento } \\
\text { brasileiro já existente }\end{array}$ \\
\hline
\end{tabular}

\section{Figura 1}

Modelo de m-payments para o mercado brasileiro

Fonte: Federação Brasileira de Bancos. (2015). Recuperado de http://www.febraban.org.br/.

A rede de dispositivos móveis tem ganhado cada vez mais espaço nos serviços de pagamentos e esse sistema de pagamento apresenta várias vantagens para as empresas e os usuários, quando comparados aos sistemas de pagamento alternativos em e-commerce. Entre as vantagens, pode-se citar o aumento da versatilidade, considerando o grande número de telefones móveis, transações mais rápidas, maior conveniência existente e economia de tempo e redução de custos (Liébana-Cabanillas, Sánchez-Fernández \& Muñoz-Leiva, 2014).

\subsection{Pagamento móvel no sistema público de transporte}

Com o aumento cada vez maior de smartphones, as pessoas utilizam cada vez mais as funcionalidades oferecidas para suas atividades diárias, como é o caso dos pagamentos móveis (Curran, Millar \& Garvey, 2012). Tais operações são vistas por muitos como a evolução natural dos processos de pagamentos digitais, mas para alguns setores, ainda não há consenso entre as partes interessadas e envolvidas, 
como é o caso do pagamento móvel para transporte público. O grande desafio a ser enfrentado são as decisões relativas à tecnologia sem fio para sua implementação, haja vista que a escolha da tecnologia apropriada é fator crucial, a fim de oferecer um esquema de pagamento satisfatório para os consumidores (Ondrus \& Pigneur, 2007).

Os pagamentos móveis são considerados como um novo canal que poderia fazer cobrança de tarifa de maneira mais conveniente. Com vistas às experiências realizadas no Japão, algumas partes interessadas já reconhecem a possibilidade de inovar os sistemas de pagamento no setor público de transportes (Ondrus \& Pigneur, 2007).

Atualmente as empresas têm testado novos sistemas de pagamento, como, por exemplo, o Near Field Communication (NFC), que é um cartão inteligente, sem contato, e um telefone móvel. O NFC, também chamado de comunicação por campo de proximidade, segundo Juntunen, Luukkainen e Tuunainen (2010) é uma tecnologia sem fios de curta distância, destinada principalmente para telefones celulares e tem muito potencial para os serviços móveis. Uma das suas aplicações mais promissoras é o mobile ticketing, que permite que telefones celulares possam ser usados no pagamento de transporte público e já tem ido utilizado em muitos países.

Uma questão que tem sido constantemente citada como a razão por trás lenta implantação NFC tem sido a limitada disponibilidade de aparelhos NFC, pois existem apenas alguns modelos de aparelhos disponíveis. Os autores afirmam que existem várias razões para a falta de aparelhos NFC no mercado. Em primeiro lugar, a normalização de certa chave de protocolos levou algum tempo e telemóveis que suportem estes padrões não puderam ser produzidos, por um tempo. Em particular, o Protocolo de Single-Wire (SWP, ETSI TS 102 613), que especifica a interface para a comunicação de baixo nível entre o Cartão de Circuito Integrado Universal e o chip NFC, só recentemente foi incorporado em projetos de aparelhos; em segundo lugar, a procura por aparelhos com essa tecnologia tem sido muito pouca, há poucos serviços NFC porque há poucos aparelhos disponíveis. Por outro lado, há pouca demanda do usuário final por aparelhos NFC, porque existem muito poucos serviços NFC. A terceira razão para a implantação lenta de NFC é que os modelos de negócios por trás dos serviços NFC não são claros, uma vez que as operadoras de 
redes móveis que subsidiam aparelhos em muitos mercados são relutantes em ordenar os dispositivos dos fabricantes sem uma visão clara de como seus investimentos serão recuperados (Juntunen, Luukkainen \& Tuunainen, 2010).

A principal vantagem da NFC em comparação a outras tecnologias sem fio é a sua simplicidade. O pagamento usando o telefone móvel com tecnologia NFC permite compras seguras e convenientes em uma ampla gama de transações, na qual encontra uma enorme aplicação em redes de sensores sem fio e vários outros campos (Nagashree, Vibha \& Aswini, 2014). A Figura 2 exemplifica um tipo de pagamento por meio de NFC.

\section{Figura 2}

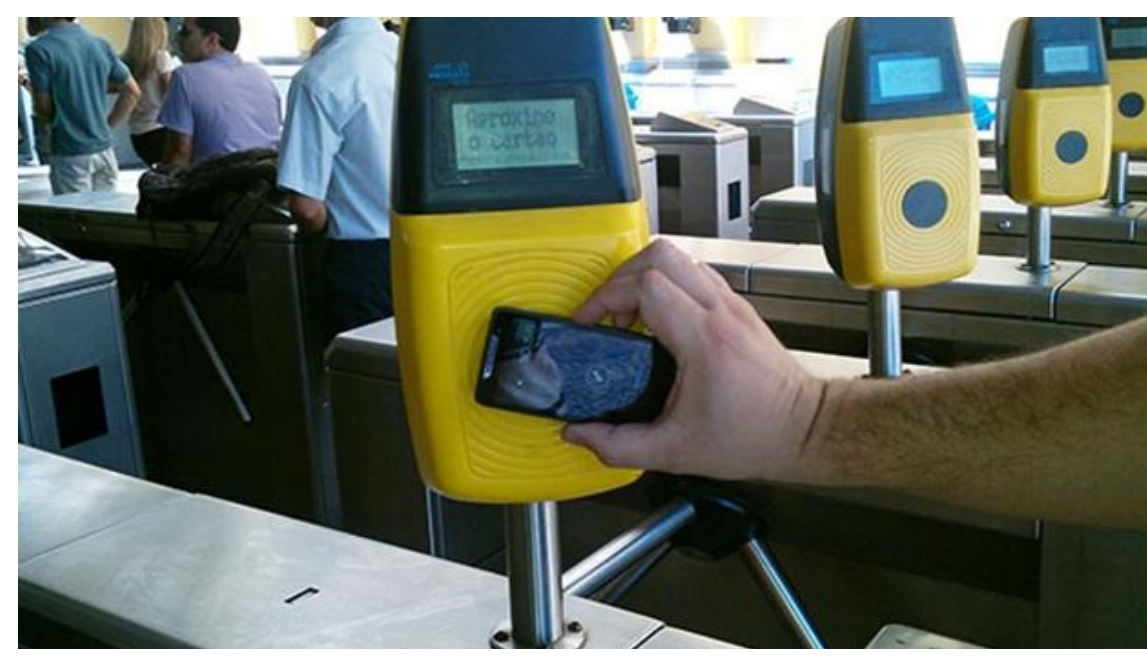

Mobile payment no transporte público através do Near Field Communication (NFC). Fonte: Rede Ponto Certo. (2015). Recuperado de http://www.redepontocerto.com.br/rpc/appRPC.jsp.

Há uma infinidade de aplicações para o sistema NFC, entre as quais pode-se citar: utilização para pagamentos móveis; bilhetagem eletrônica; serviços baseados em localização; compras, em substituição ao código de barras; saúde e aplicações médicas; transferência de dados em geral; identificação, (passaportes e documentos de identificação eletrônicos já estão sendo emitidos em muitos países). Enquanto muitas aplicações NFC podem funcionar perfeitamente bem sem armazenar informações confidenciais no dispositivo móvel, outros aplicativos, como o pagamento e emissão de bilhetes, precisam lidar com dados confidenciais e requerem um local de armazenamento seguro para obter essas informações (Curran, Millar, \& Garvey, 2012).

Há estimativas de crescimento de mercado dos dispositivos com tecnologia NFC, e em consequência disso, há uma preocupação com a questão da segurança. 
O intervalo NFC é de apenas alguns centímetros, fazendo com que seja mais seguro do que as tecnologias de maior alcance existentes; porém, há ainda falhas de segurança que precisam ser sanadas (Curran, Millar \& Garvey, 2012).

\section{Método da produção científica}

O objetivo desse relato foi apresentar o sistema de pagamento móvel no transporte público da cidade de São Paulo e por que esse sistema ainda é tão pouco utilizado pelos usuários.

Esse estudo tem abordagem qualitativa e a estratégia de pesquisa adotada é o estudo de caso único, que segundo Yin (2015), permite ao investigador um aprofundamento em relação ao fenômeno estudado, favorecendo uma visão holística sobre os acontecimentos da vida real, destacando seu caráter de investigação empírica de fenômenos contemporâneos.

Para fins de coleta de dados, as técnicas de pesquisa utilizadas foram observação não participante, na qual os autores presenciaram o fato, mas não tiveram participação efetiva no projeto desenvolvido e segundo Marconi e Lakatos (2010), o observador não interage com o objeto de estudo no momento em que realiza a observação, e o processo de coleta de dados ocorre no ambiente do objeto de estudo; e a pesquisa bibliográfica utilizada como fontes secundárias, que segundo Martins e Theófilo (2009, p. 54), "busca conhecer, analisar e explicar contribuições sobre determinado assunto, tema ou problema".

\section{Contextualização do Projeto}

\subsection{Caracterização da organização}

Segundo dados da SPTrans (2015), a empresa foi fundada em 1995, tendo por finalidade a gestão do sistema de transporte da cidade de São Paulo. O Sistema Municipal de Transporte é composto por uma rede integrada, criada pela Secretaria Municipal de Transportes, em conjunto com a SPTrans, rede essa que permite um deslocamento mais rápido e a racionalização do uso dos meios de transporte na cidade. Esta rede integrada é formada por empresas de ônibus, pela Companhia do 
Metropolitano (Metrô), e pela Companhia Paulista de Trens Metropolitanos (CPTM). Estas duas últimas são controladas pelo Governo Estadual.

O Metrô de São Paulo possui cinco linhas em operação; ao todo são 69,4 quilômetros de rede, 61 estações e 150 trens. O sistema é integrado à CPTM em algumas estações e transporta em média 4,7 milhões de passageiros por dia (METRÔ, 2015). Já a CPTM possui 6 linhas, numa malha total de 260,8 quilômetros, atendendo 22 municípios - sendo 19 deles na Região Metropolitana de São Paulo - e 92 estações e são transportados em média 2,8 milhões de passageiros por dia (CPTM, 2015). As linhas de ônibus são operadas por 16 consórcios e formados por empresas e cooperativas e atualmente conta com uma frota de aproximadamente 15.000 ônibus (SPTRANS, 2015).

Já para ligar a região metropolitana de São Paulo às demais regiões da cidade, conta-se com o sistema metropolitano de ônibus, sob responsabilidade da EMTU, subordinada ao Governo Estadual. Na região metropolitana da cidade de São Paulo, aproximadamente $55 \%$ das viagens motorizadas são feitas em transporte coletivo, totalizando 6 milhões de passageiros transportados por dia útil (SPTRANS, 2015).

\subsection{Caracterização do projeto}

Desde julho de 2014 foi implementado o sistema Mobile para recarga de cartão para transporte público dos usuários do transporte público de São Paulo. Inicialmente o aplicativo foi disponibilizado apenas para celulares com tecnologia NFC (Near Field Communication) ou Comunicação por Campo de Proximidade para todos os aparelhos com Android (4.1 ou superior) e IOS (8.3 ou superior), que realizam a transferência de dados de um dispositivo para outro, bastando encostar o cartão na parte de trás do aparelho para fazer o pareamento das informações. $O$ app em questão foi desenvolvido pela Rede Ponto Certo, empresa que controla parte dos quiosques de recarga instalados na capital paulista, e os aparelhos com a tecnologia NFC tem as funções de consultar saldos, recarregar vale transporte, créditos e comprar créditos de Passe Comum, Estudante, Mensal, Semanal e Diário. Já para os aparelhos que não possuem a tecnologia NFC, o aplicativo foi disponibilizado posteriormente, sendo possível somente comprar créditos de Passe Comum, Estudante, Mensal, Semanal e Diário (SPTRANS, 2015). Segundo $@$ Revista Gestão \& Tecnologia, Pedro Leopoldo, v. 17, n. 1, p. 258-276, jan./abr. 2017 
informações da Rede Ponto Certo (2015), o sistema é bem simples, podendo ser baixado pela loja virtual da Google Play. A Figura 3 ilustra como funciona o app móbile Rede Ponto Certo.

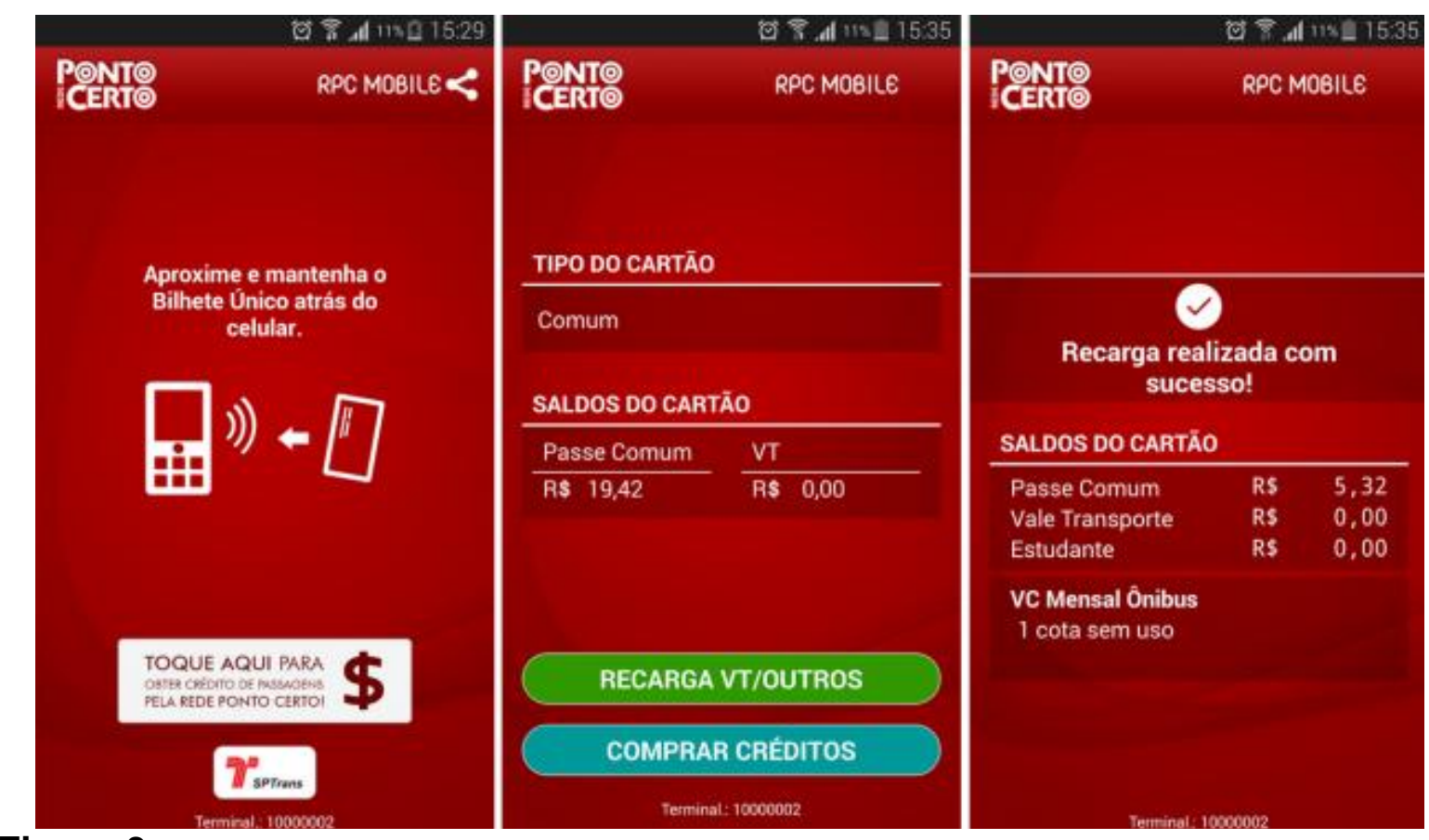

Figura 3

App mobile Rede Ponto Certo

Fonte: Rede Ponto Certo. (2015). Recuperado de http://www.redepontocerto.com.br/rpc/appRPC.jsp.

Para a instalação da ferramenta, é preciso entrar no Google Play, procurar por Ponto Certo Bilhete Único; depois de instalado, é preciso informar o CPF do usuário, encostar o Bilhete Único na parte de trás do celular. O saldo aparecerá, e também as opções de recarga e compra de créditos. Para a compra e/ou recarga de créditos, basta clicar em compra de créditos, informar o valor da recarga e a forma de pagamento - podendo ser através de boleto bancário, cartões Visa Débito ou MasterCard Débito - efetuar o pagamento e aguardar a confirmação. É preciso encostar o Bilhete Único novamente através do celular para validar os créditos (Rede Ponto Certo, 2015).

O novo sistema faz com que o celular seja um posto de recarga portátil, que se junta a uma rede de 10 mil postos disponíveis aos usuários, com a vantagem de que ele pode ser levado para qualquer lugar. Atualmente, o aplicativo oficial para tais fins é somente o que foi desenvolvido pela Rede Ponto Certo, mas outras empresas credenciadas também poderão desenvolver (SPTRANS, 2015). 
Em novembro de 2015 foi lançado o Watch2pay (Figura 4), um relógio que possui um chip que funciona somente para Bilhete Único Comum da SPTrans e dará acesso somente ao transporte em São Paulo (Capital).
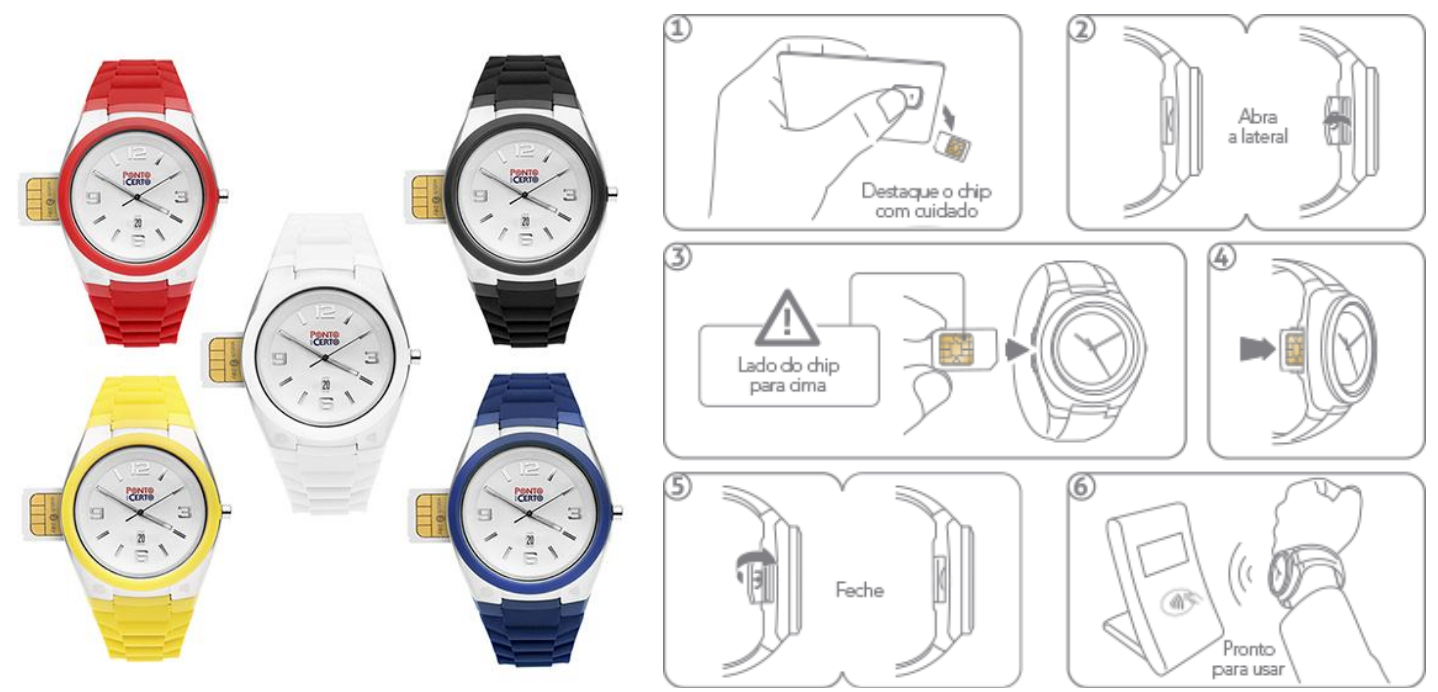

\section{Figura 4}

Watch2Pay da Rede Ponto Certo

Fonte: Rede Ponto Certo. (2015). Recuperado de http://www.redepontocerto.com.br/rpc/appRPC.jsp.

O relógio é comercializado pelo valor de $R \$ 249,00$ e não vem com créditos disponíveis, sendo necessário carregá-lo após a compra.

\section{Resultados Obtidos e Análise}

Como descrito no item 3 do presente relato, não houve participação dos pesquisadores no desenvolvimento e implantação do projeto em questão; portanto, não houve intervenção. O mecanismo aqui adotado é apenas o de relatar e apresentar como se dá o funcionamento do sistema de pagamento móvel adotado pela empresa estudada e as principais falhas/lacunas nele identificadas.

Desde julho de 2014, os usuários do transporte público de São Paulo contam com um aplicativo Mobile para compra e recarga de cartão. Os pagamentos móveis vêm ganhando cada vez mais adeptos no mundo corporativo, e o transporte público é um deles. As empresas têm procurado inovações e adequações a esta tecnologia móvel. No entanto, o projeto implementado através da Rede Ponto Certo para o transporte público da cidade de São Paulo é que a tecnologia utilizada para a recarga do bilhete único através do pagamento móvel - NFC - é disponível apenas em aparelhos com sistema operacional Android, e para alguns modelos de 
aparelhos, pois outros, mesmo tendo sistema operacional Android, não possuem tecnologia NFC. Dessa forma, a utilização do sistema implementado não é acessível a todos os usuários, sendo contrária à opinião de Battard e Mangematin (2013), que explicam que as tecnologias móveis têm o objetivo de facilitar e melhorar a vida das pessoas, permitindo que elas possam se conectar em qualquer lugar e a qualquer momento, devendo seu uso ser conveniente, flexível e democrático.

Segundo informações da empresa, cerca de 2,5 milhões de pessoas podem ser beneficiadas com a novidade. Para uma cidade que, segundo dados da própria SPTrans (2015), transporta em média aproximadamente 10 milhões de passageiros/dia útil, esse número é muito pequeno. A quantidade de aparelhos que tem disponibilidade para o sistema operacional NFC também é muito baixa, levando em consideração a quantidade de novos modelos que são anunciados ou lançados todos os meses no mercado.

Devem ser levados em consideração alguns fatores relevantes, como: 1) o perfil do usuário dominante no transporte público pertence às classes $C, D$ e $E$, pois segundo Araújo et al. (2011), a mobilidade individual da classe A e B na cidade de São Paulo é o dobro da classe D e E; 2) segundo dados da empresa Kantar Worldpanel (2015), em 2013, o uso de smartphones das classes C, D e E era de 29\%, ou seja, a quantidade de usuários que possuem um aparelho com a tecnologia disponível ainda é muito pequena, uma vez que também devemos levar em consideração o fato de que nem todos os smartphones possuem a tecnologia NFC; 3) é justamente esse usuário que pertence à categoria não bancarizada, ou seja, que não possui conta e acesso aos serviços bancários, e que, segundo dados da Febraban (2015), representam 39,5\% da população adulta, e a compra e recarga de cartões no sistema implementado é através do uso de cartão de débito, transferência bancária (apenas dois bancos disponibilizam esse serviço), ou boleto bancário.

Com relação ao Watch2pay, o relógio seria muito viável levando em consideração que grandes operadoras, tanto de cartão de crédito quanto de telefonia e os próprios bancos ainda não incluíram em seus serviços pagamento por celular. No entanto, o relógio possui um preço nada convidativo, e para a grande maioria da população usuária do transporte público de São Paulo, que é pertencente às classes econômicas menos favorecidas, o uso dessa tecnologia dificilmente irá emplacar. 
A falta de divulgação desse novo serviço pode ser considerada deficiente, pois durante o processo de elaboração deste relato, não foram observados sinalização, folder ou até mesmo divulgação de mídia digital dentro dos trens e/ou ônibus ou nas redes sociais sobre o sistema em pauta. Isso é contrário ao que argumenta Amaral (2008), pois a divulgação do serviço é fundamental para o conhecimento e adoção do sistema por parte do usuário, sendo uma atividade que tem o propósito de possibilitar que o usuário conheça, efetive e adote um produto ou serviço, tornando-os usuários potenciais, fazendo com que o serviço seja atraente a esse usuário, bem como apresentar e evidenciar seus benefícios e vantagens.

As filas são um problema constante para quem necessita comprar ou recarregar o seu bilhete, não sendo raros os dias em que o sistema está lento, ou muitas vezes inoperante; sendo assim, o usuário perde muito do seu tempo nas filas, conforme Figura 5.

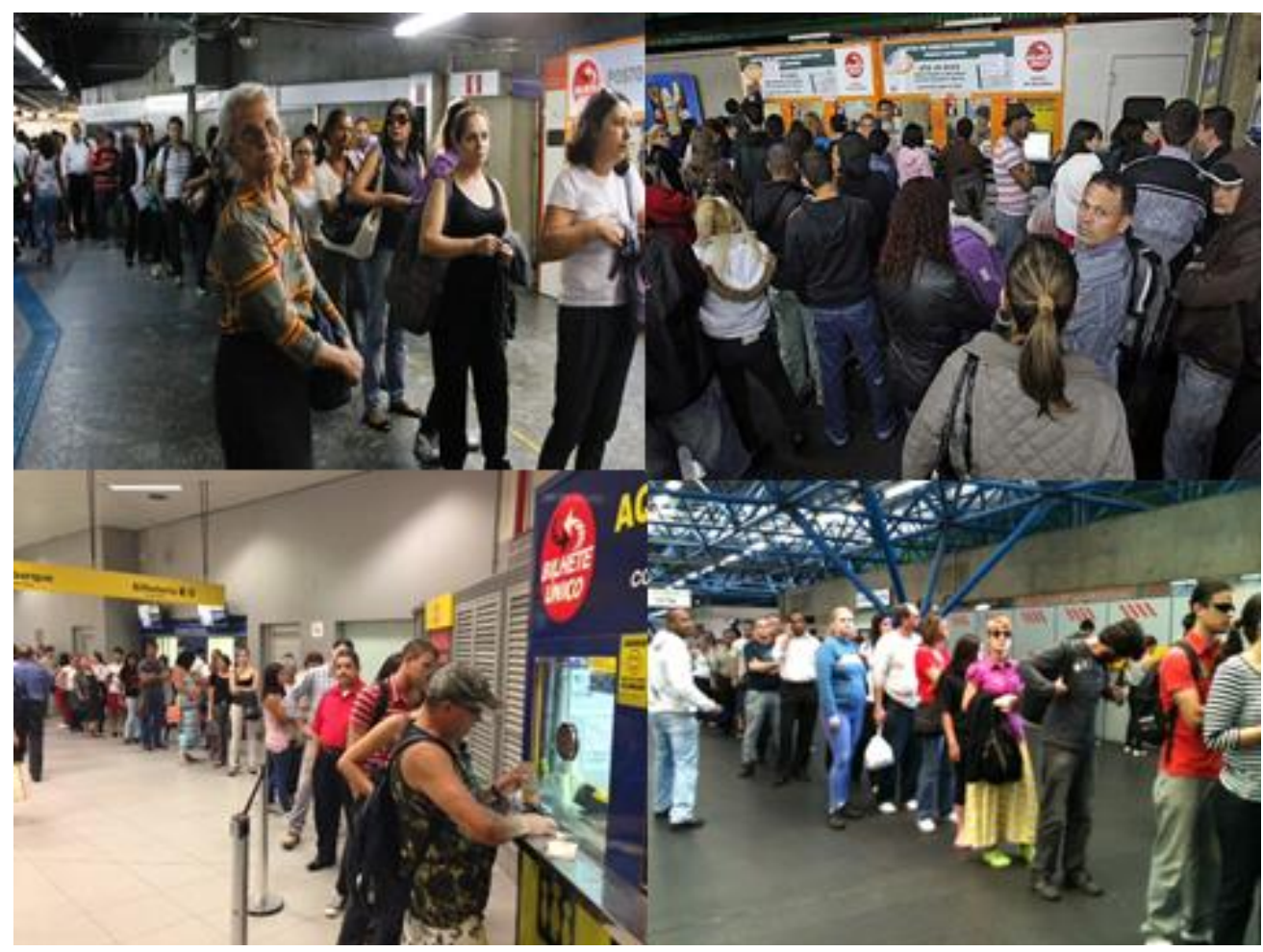

Figura 5

Filas nos guichês de recarrega do bilhete único em diferentes estações de metrô em São Paulo

Fonte: Pinho, M. Recarregar bilhete único nesta segunda 'congela' tarifa em $\mathrm{R} \$ 3$. Recuperado de http://g1.globo.com/sao-paulo/noticia/2015/01/-3.html

A eficiência no sistema implementado reduziria as filas nos postos de compra e recarga de bilhete, bem como não haveria a necessidade de pagar em dinheiro, 
sendo que muitas vezes o usuário não dispõe de dinheiro na carteira. Um sistema de pagamento móvel abarca um ecossistema e há grandes barreiras e complexidades no processo, pois conforme opinião de Ondrus e Pigneur (2006) envolve usuários, prestadores de serviços e instituições financeiras. O sistema implementado de pagamento móvel para transporte público em São Paulo ainda é uma tarefa inicial e complexa, que compreende um ecossistema que abrange usuários, o sistema SPTrans, operadora do sistema de cartão, os fabricantes de smartphones, empresas de serviços financeiros, fornecedores de serviços, dispositivos, software e o Governo, uma vez que duas das empresas que fazem parte do sistema SPTrans são administradas pelo Governo Estadual; isso requer boa vontade e engajamento de ambas as partes para que o sistema realmente funcione de maneira que satisfaça e seja de utilidade para o usuário, cumprindo assim o seu objetivo.

\section{Conclusão}

Os sistemas de comunicações móveis revolucionaram a forma como as pessoas se comunicam, unindo comunicação e mobilidade, e com o passar dos anos, o acesso através da tecnologia sem fio teve grandes evoluções relacionadas ao desempenho e eficiência. A mobilidade tecnológica é uma revolução no cotidiano das pessoas, pois através de dispositivos móveis é possível a utilização de diversos serviços enquanto estiver em movimento, em qualquer lugar e a qualquer momento, acessibilidade e conveniência, além de conectividade instantânea.

A utilização de dispositivos móveis com a tecnologia NFC proporcionam inúmeros benefícios, entre eles, como visto no decorrer deste trabalho, está a utilização para pagamento móvel e transferência de informações seguras. Tal método de pagamento tem permitido aos usuários, além da facilidade de uso e velocidade nas operações, eliminar o pagamento com dinheiro. No entanto, apesar de toda a facilidade que essa tecnologia oferece, depende da disponibilidade, confiabilidade e aceitação dos sistemas de pagamento móvel. Os dispositivos móveis equipados com sistema NFC, de uma forma geral, são considerados rápidos e confiáveis.

Em diversos países, o uso de dispositivos móveis para pagamentos em transportes públicos já é uma realidade e estão cada vez mais em alta, em troca dos 
sistemas comumente conhecidos, que são a compra e/ou recarga de bilhete nos guichês das estações. A mobilidade tecnológica, na opinião de Machado e Freitas (2007), gera impactos sociais a partir do momento em que os usuários se apropriam de seus atributos e funcionalidades, adotando-as de diferentes maneiras e passem a fazer cada vez mais parte de seu cotidiano, mudando até mesmo a forma como os indivíduis interagem. O sistema de pagamento móvel implementado pela Rede Ponto Certo para o transporte público na cidade de São Paulo é uma novidade que facilita o dia-a-dia dos usuários, porém, há deficiências ainda a serem sanadas conforme já descrito - para que torne um serviço atrativo e que incentive o cliente a adotá-lo.

As limitações percebidas na elaboração desse trabalho foram as poucas informações disponíveis e também a falta de acessibilidade aos dados da empresa, ao processo de desenvolvimento e à implantação do projeto. Dentro do que foi possível investigar e estudar, com relação à apresentação do sistema implementado, o presente relato atingiu o seu objetivo.

Para trabalhos futuros, pretende-se aprofundar o estudo e analisar outros possíveis aspectos que estejam colaborando para a baixa utilização do sistema, através de uma pesquisa com os usuários, para conhecer sua opinião e percepção quanto ao sistema implementado, bem como fazer um comparativo entre o sistema na cidade de São Paulo e outras cidades e países.

\section{Referências}

Amaral, S.A. (2008) Marketing da informação: entre a promoção e a comunicação integrada de marketing. Informação \& Sociedade: Estudos, 18(1), 31-44.

Amoroso, D. L., \& Watanabe, R. M. (2012). Building a research model for mobile wallet consumer adoption: the case of mobile Suica in Japan. Journal of Theoretical and Applied Electronic Commerce Research, 7(1), 94-110.

Araújo, M. R. M., Oliveira, J. M., Jesus, M. S., Sá, N. R., Santos, P. A. C., \& Lima, T. C. (2011). Transporte público coletivo: discutindo acessibilidade, mobilidade e qualidade de vida. Psicologia \& Sociedade, 23(3).

Battard, N., \& Mangematin, V. (2013). Idiosyncratic distances: Impact of mobile technology practices on role segmentation and integration. Technological Forecasting \& Social Change, 80, 231-242.

Carr, M. (2007). Mobile payment systems and services: an introduction. Recuperado de 
http://www.academia.edu/2563249/Mobile_Payment_Systems_and_Services_An _Introduction.

Companhia Paulista de Trens Metropolitanos. (2015). Recuperado de http://www.cptm.sp.gov.br/a-companhia/Pages/Nossa-Historia.aspx.

Corso, K. B., Freitas, H. M. R., \& Behr. (2013). O contexto no trabalho móvel: uma discussão à luz do paradigma da ubiquidade. Revista Administração em Diálogo, 15(2), 01-25.

Curran, K., Millar, A., \& Garvey, C. M. (2012). Near field communication. International Journal of Electrical and Computer Engineering, 2(3), 371-382.

Federação Brasileira de Bancos - FEBRABAN. (2015). Recuperado de http://www.febraban.org.br/.

Finzgar, L., \& Trebar, M. (2011). Use of NFC and QR code identification in an electronic ticket system for public transport. Anais do Software, Telecommunications and Computer Networks (SoftCOM), Croatia, Adriatic Islands.

Ghezzi, A., Cortimiglia, M. N., \& Frank, A.G. (2015). Strategy and business model design in dynamic telecommunications industries: a study on Italian mobile network operators. Technological Forecasting \& Social Change, 90, 346-354.

Juntunen, A., Luukkainen, S., \& Tuunainen, V.K. (2010). Deploying NFC technology for mobile ticketing services - identification of critical business model issues. Mobile Business and Ninth Global Mobility Roundtable, 82-90.

Kantar Wordpanel. (2015). Recuperado de www.kantarworldpanel.com/br

Kumar, S., Liu, Y., Sengupta, J., \& Divya. (2010). Evolution of mobile wireless communication networks: $1 \mathrm{G}$ to $4 \mathrm{G}$. International Journal of Electronics \& Communication Technology, 1, 68-72.

Kumar, S. B. R. \& Rabara, S. A. (2010). An architectural design for secure mobile remote macro-payments. Jagannath Gupta Institute of Engineering \& Technology (JNIT), 1, 75-84.

Leong, L. Y., Hew, T-S., Tan, G. W-H., \& Ooi, K. B. (2013). Predicting the determinants of the NFC-enabled mobile credit card acceptance: a neural networks approach. Expert Systems with Applications, 40, 5604-5620.

Liebana-Cabanillas, F. J., Sánchez-Fernández, J., \& Munoz-Leiva, F. (2014). Role of gender on acceptance of mobile payment. Industrial Management \& Data Systems, 114(2), 220-240. 
Machado, C. B., \& Freitas, A. A. (2007). Modelo para planejamento de iniciativas de adoção de tecnologias móveis na interação entre organização e indivíduo. Anais do Encontro de Administração da Informação (EnADI), Florianópolis, SC, Brasil.

Marconi, M. A. \& Lakatos, E. V. (2001). Fundamentos de metodologia científica. São Paulo: Atlas.

Martins, G. A., \& Theófilo, C. R. (2009). Metodologia da investigação científica para ciências sociais aplicadas. São Paulo: Atlas.

Metrô. (2015). Recuperado de http://www.metro.sp.gov.br/metro/index.aspx.

Nagashree, R. N., Vibha, R., \& Aswini, N. (2014). Near field communication. I.J. Wireless and Microwave Technologies, 4(2), 20-30.

Ondrus, J., \& Pigneur, Y. (2006). Towards a holistic analysis of mobile payments: a multiple perspectives approach. Electronic Commerce Research and Applications, 5, 246-257.

Ondrus, J., \& Pigneur, Y. (2007). An assessment of NFC for future mobile payment systems. Management of Mobile Business, 9-11.

Pierre, S. (2001). Mobile computing and ubiquitous networking: concepts, technologies and challenges. Telematics and Informatics, 18(2-3), 109-131.

Pinho, M. (2015). Recarregar bilhete único nesta segunda 'congela' tarifa em $R \$ 3$. de http://g1.globo.com/sao-paulo/noticia/2015/01/recarregar-bilhete-unico-nestasegunda-congela-tarifa-em-r-3.html

Rede Ponto Certo. (2015). Recuperado de http://www.redepontocerto.com.br/rpc/appRPC.jsp.

Saccol, A. Z., \& Reinhard, N. (2007). Tecnologias de informação móveis, sem fio e ubíquas: definições, estado-da-arte e oportunidades de pesquisa. Revista Administração Contemporânea, 11(4), 175-198.

Saha, D., \& Mukherjee, C. A. (2003). Pervasive computing: a paradigm for the 21st century. IEEE Computer Society, 25-31.

Sheng, H., Nah, F. F. H., \& Siau, K. (2005). Strategic implications of mobile technology: a case study using value-focused thinking. Journal of Strategic Information Systems, 14, 269-290.

SPTrans. (2015). Recuperado de http://www.sptrans.com.br/a_sptrans/.

Yin, R. K. (2015). Estudo de caso planejamento e métodos. Porto Alegre: Bookman. 